\title{
Supporting Secure Spectrum Sensing data Transmission against SSDH Attack in Cognitive Radio Ad hoc Networks
}

\author{
Jingyu Feng ${ }^{1,2}$, Guangyue $\mathrm{Lu}^{1 *}$, Honggang Wang ${ }^{1}$ and Xuanhong Wang ${ }^{1}$ \\ 1. Department of Communication Engineering, Xi'an University of Posts \& Telecommunications, Xi'an 710121, China \\ 2. State Key Laboratory of Information Security (Institute of Information Engineering), Chinese Academy of Sciences, \\ Beijing 100093, China
}

\begin{abstract}
Cognitive radio ad hoc networks are commonly perceived as ideal ad hoc environments where cognitive radio technology enables secondary users (SUs) to utilize scarce spectrum resources in a dynamic manner. Cooperative spectrum sensing (CSS) is the key function of cognitive radio technology to identify the available spectrum. However, the nature of aggregating data makes CSS offer opportunities for malicious SUs. Recently, a lot of efforts have been paid to combating spectrum sensing data falsification (SSDF) threat, but little attention to the multi-hop architecture of cognitive radio ad hoc networks. In this paper, we report the discovery of a novel attack called spectrum sensing data hijack (SSDH), in which attackers disguise as routers to hijack and tamper with spectrum sensing data during the transmission. Our simulations show that this new attack needs much less cost to manipulate CSS and has a much higher success rate compared with SSDF attack. We conduct an in-depth investigation on SSDH and propose a two-level defense scheme from the design ideas of IBC signature-verification and neighbor monitor. We also perform simulations to validate our approach. The results show that our defense scheme can significantly reduce the SSDH attack success ratio.
\end{abstract}

Keywords Cognitive radio, ad hoc, cooperative spectrum sensing, hijack, trust.

\section{Introduction}

With the rapid development of wireless communication and the huge demand of the capacity for wireless applications, the wireless frequency spectrum has become increasingly scarce. At the same time, a large portion of the assigned spectrum bands, such as in the $400-700 \mathrm{MHz}$ range, that are used sporadically or under-utilized for transmission [1]. To solve the contradiction between the spectrum scarcity and low spectrum utilization, cognitive radio has been considered as a useful technology. Currently, cognitive radio technology is introduced in ad hoc networks, and thus forming cognitive radio ad hoc networks (CRAHNs) which refer to the distributed networks where unlicensed user (or Secondary User-SU) can share the spectrum with licensed user (or Primary User-PU) if they do not cause any interference to PU [2]. CRAHNs are gaining importance with the increasing number of potential applications, such as military battlefield communications, disaster relief, and autonomous vehicular communications [3].

Cooperative spectrum sensing (CSS) is the key function of cognitive radio technology to enhance the detection performance by exploiting spatial diversity via the observations of spatially located SUs. In a CSS architecture, all the participating SUs forward their observations regarding the presence or absence of a PU to a fusion center (FC), which makes the final decision about whether the PU is transmitting or not. This nature of aggregating data makes CSS offer opportunities for malicious SUs to launch SSDF [4] attack by sending false spectrum sensing data. For convenience, spectrum sensing data are abbreviated as sensing data in the rest of this paper.

*Corresponding author: Guangyue Lu, E-mail: tonylugy@163.com

This research was supported in part by the National Science Foundation of China (61301091, 61271276), the Open Foundation of State Key Laboratory of Information Security (2015-MS-14), the Industrial Science and Technology project of Shaanxi Province (2014K05-09, 2015GY013), the Natural Science Foundation of Education Department of Shaanxi Province (14JK1660), the New Star Team of Xi'an University of Posts \& Telecommunications. 
Fortunately, SSDF attack can be suppressed by trust mechanism and many efforts have been made to study various trust mechanisms [5-8]. They estimate whether an SU is trustworthy or not by its past behaviors and give low weights to the sensing data from less trustworthy SUs when generating a final decision. In addition, it may be a huge task for malicious SUs to launch SSDF attack since a malicious SU can only fake one sensing datum at each CSS action. That is, to change the final decision from CSS, a sufficient number of malicious SUs are necessary.

Note that CRAHN protocols follow message forwarding mechanisms in CSS, where the SUs with strong report channel can serve as relays to assist in forwarding sensing data from the SUs with weak report channel [9]. In this paper, we discover a novel attack along this line, named as spectrum sensing data hijack (SSDH), against CSS in CRAHNs, and propose a two-level defense scheme called GSSD to defend against this attack. The main contributions of this paper are as following:

- Conduct an in-depth investigation on SSDH attack, including four types of threats, attack strategy and attack power. Compared with SSDF attack, SSDH needs less cost to achieve the similar goals. The basic idea of SSDH is as follows. SSDH attackers disguise as routers to hijack and tamper with sensing data from the SUs who can sense PU singal. As a result, the SUs who require sensing data will make a wrong final decision, but the SUs who sensed PU singal may be considered as dishonest and their trustworthniess will be reduced since their sensing data disagree with PU's actual status. Nevertheless, SSDH attackers get away with punishment.

- Use IBC signature-verification as the first level defense scheme. It is difficult to adopt the public key cryptography (PKC) to encrypt or sign sensing data since no a central authority can be employed to manage key exchange. We design the first level defense scheme based on the identity-based cryptography (IBC) [10]. This scheme asks the SUs who can sense PU singal to sign sensing data with their private keys and produce a digital signature. This signature confirms the authenticity and integrity of sensing data, and thus increasing the difficulty of launching SSDH attack.

- Introduce the design idea of neighbor monitor as the second level defense scheme. Three advantages can be found in the second level defense scheme: identifing tampered sensing data, correcting sensing data and isolating SSDH attackers. To identify tampered sensing data, the first level defense scheme is performed by each router (such as $\mathrm{SU}_{i}$ ) to monitor his previous router (such as $\mathrm{SU}_{p}$ ). If tampered, the neighbor tie value $n t_{i p}$ generated from $\mathrm{SU}_{i}$ to $\mathrm{SU}_{p}$ will be reduced. Considering that one sensing datum is a binary variable $\{0,1\}$, we ask that the behavior of forwarding tampered sensing data will also cause the decrease of $n t_{i p}$ for $\mathrm{SU}_{p}$. This stimulates $\mathrm{SU}_{p}$ corrects sensing data when finding his neighbors tampered with these data. By neighbor monitor, SSDH attackers will get bad neighbor tie value from his neighbors who have the right to refuse to forward SSDH attackers' CSS help query. In this case, SSDH attackers can be isolated from CSS.

\section{Preliminaries}

\subsection{Cooperative spectrum sensing}

In the CRAHNs, SUs cooperate with each other to achieve a CSS exchange in the self-organizing manner due to the lack of centralized control. As shown in Fig.1, when an initiator SU (such as $\mathrm{SU}_{0}$ ) wants to know the spectrum status of the target PU in a CRAHN, a CSS exchange will be trigged: individual sensing, data reporting and data fusion.

- Individual sensing: Each SU senses the vacant spectrum of the PU via the sensing channel individually. The sensing channel is the selected licensed frequency band where a physical point-to-point link between the PU transmitter and each cooperating SU for observing the primary spectrum [9].

- Data reporting: All SUs send their sensing data to $\mathrm{SU}_{0}$ via the report channel. The reporting channel is a control channel where a physical point-to-point link between each cooperating SU and the initiator SU for sending individual sensing information [9]. If both sensing channel and reporting channel are not perfect, an SU observing a weak sensing channel and a strong report 
channel and another SU with a strong sensing channel and a weak reporting channel, for example, can complement and cooperate with each other to improve the performance of CSS. In Fig.1, $\mathrm{SU}_{3}$ and $\mathrm{SU}_{4}$, who observe strong PU signals, may suffer from a weak reporting channel. $\mathrm{SU}_{1}, \mathrm{SU}_{5}$ and $\mathrm{SU}_{6}$, who have a strong reporting channel, can serve as relays to assist in forwarding the sensing data from $\mathrm{SU}_{3}$ and $\mathrm{SU}_{4}$ to $\mathrm{SU}_{0}$.

- Data fusion: Without a central authority, $\mathrm{SU}_{0}$ combines the received sensing data and to determine the final decision of PU spectrum. The final decision are usually made according to three typical CSS fusion rules, such as the "AND", "OR" and "Majority" rule [11].

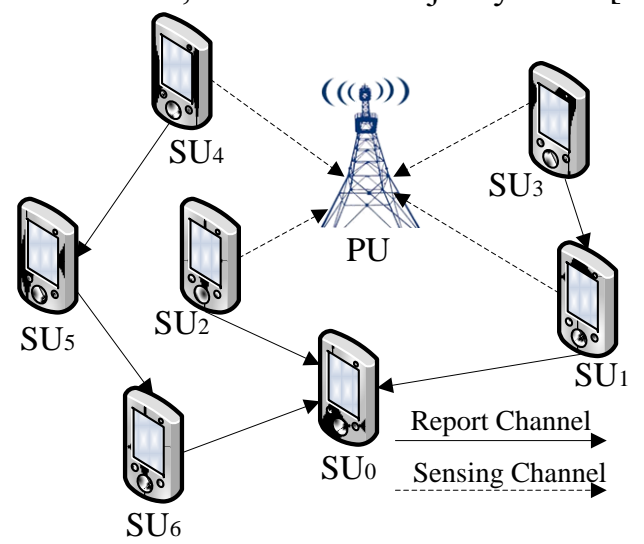

Fig. 1 The CSS exchange launched by $\mathrm{SU}_{0}$ in a CRAHN .

Typically, individual sensing for primary signal energy detection can be formulated as a binary hypothesis problem as follows [3]:

$$
y(t)= \begin{cases}n(t), & H_{0} \\ h(t) \cdot s(t)+n(t), & H_{1}\end{cases}
$$

where $y(t)$ represents the detected signal at each SU, $s(t)$ is the transmitted PU signal, $h(t)$ is the channel gain of the sensing channel, $n(t)$ is the zero-mean additive white Gaussian noise (AWGN), $H_{0}$ and $H_{1}$ denote the hypothesis of the absence and the presence of the PU signal, respectively. If the estimated energy of the received signal is larger than the decision threshold, the existence of PU would be declared. Otherwise, if the energy of the received signal is smaller than the threshold, there is no PU signal.

After the individual sensing, the individual sensing data of each SU is determined. $d_{i}$ indicates the individual sensing data of $\mathrm{SU}_{i}$, which is usually expressed as a binary variable:

$$
d_{i}= \begin{cases}0, & H_{0} \\ 1, & H_{1}\end{cases}
$$

where " 0 " and " 1 " denote the hypothesis of the absence and the presence of the PU singal, respectively. The spectrum sensing problem therefore can be regarded as a binary. Correspondingly, the final decision $d$ is also binary under the "AND", "OR" and "Majority" rule. In the "AND" rule, FC determines $d=1$ if all individual sensing $d_{i}=1$. The "OR" rule refers to $d=1$ if an individual sensing $d_{i}=1$. The "Majority" rule requires at least a half of SUs to report 1. The "OR" rule works best when the number of SUs is large, whereas the "AND" rule works well when the number of cooperating users is small, and the "Majority" rule can be obtained from the $k$ out of $N$ rule under the condition when $k \geq N / 2$ [9].

\subsection{Trust mechanism}

Trust mechanism is increasing influence on many application scenarios, including e-commerce [12], wireless sensor networks [13], ad hoc routing [14], online social networks [15], internet of things [16], etc.

Trust mechanism also plays significant roles in CSS area, such as 1) assisting an initiator SU' accurate decision-making, 2) encouraging honest behaviors, and 3) preventing participating from malicious SUs. 
Representative trust mechanisms are as follows. In [5], the authors propose a novel trust-aware hybrid spectrum sensing scheme, in which the Beta reputation is used to construct trust scheme. Zeng et al propose a reliable CSS scheme with trusted SUs assistance for mitigating SSDF attack in [6]. In [7], the authors consider trust as a competitive factor to punish malicious SUs to access any vacant PU spectrum. In [8], the authors measure the trust of SUs in CSS during the cognition cycle, and incorporate it into the sensing data fusion to reduce the effect of malicious SUs on final decision making. The common property of these existing trust mechanism schemes is that the trustworthiness of an SU is evaluated by his historical sensing behaviors low weights are given to the sensing data from less trustworthy SUs when generating a final decision. However, they construct trust mechanism in central environments, so these schemes cannot be applied to CRAHNs directly.

We find that SSDH attack will reduce or hurt the trustworthiness of the SUs whose honest sensing data are tampered as false. A basic trust mechanism in CRAHNs is described, which will be used to analyze how SSDH attack can influence the calculation of trust value in Section 5. Generally, the CRAHN is a small-scale network, and the data size generated by historical CSS exchange is limited. Without the help of central authority, an initiator $\mathrm{SU}$ (such as $\mathrm{SU}_{i}$ ) can record the historical sensing data of the SUs who have cooperated with him for detecting the target PU, and consult the actual spectrum status (ass) of the target PU to update the trust value of these $\mathrm{SUs}$. For $\mathrm{SU}_{k}$, his behavior value $\left(h_{i k}, f_{i k}\right)$ observed at $\mathrm{SU}_{i}$ after each CSS exchange can be updated as

$$
\text { behavior value } \stackrel{\text { update }}{\longrightarrow} \mid \begin{aligned}
& h_{i k}=h_{i k}+1, \text { if } d_{k} \text { agrees with ass } \\
& f_{i k}=f_{i k}+1 \text {, if } d_{k} \text { disagrees with ass }
\end{aligned}
$$

Then, the trust value of $\mathrm{SU}_{k}$ at $\mathrm{SU}_{i}$ can be calculated as

$$
T_{i k}=\frac{h_{i k}}{h_{i k}+f_{i k}}
$$

\section{SSDH attack overview}

Since the individual sensing report is usually regarded as a binary variable, it is very easy for malicious SUs to take advantage of CSS and launch SSDF attack by faking individual spectrum sensing data, resulting in a wrong final sensing decision.

Generally speaking, the basic goal of SSDF attackers against CSS is to illegally occupy or disturb the PU spectrum bands. Such attackers can be classified according to their attack intention [17].

- Always-free: The attackers submit an absent licensed signal, although there are PUs using their spectrums. In this case the initiator SU makes a wrong decision that the PU spectrums are free and will use them. The intention of such attackers is to give interference to PUs.

- Always-busy: The attackers declare that the licensed user is active, although there are no PU singals. In this case the initiator $\mathrm{SU}$ (such as $\mathrm{SU}_{0}$ ) makes a wrong decision that PUs are present and will not use the spectrum. The intention of such attackers is to gain exclusive access to the target spectrum.

These two types of SSDF attackers are dangerous. Fortunately, they can be easily detected by current trust mechanism if malicious SUs always report false sensing data. This is because they will obtain a lower trust value when they always submit false sensing data. In addition, it may be a huge task or malicious SUs to launch SSDF attack since a malicious SU can only fake one sensing datum at each CSS action. These questions lead to the discovery of SSDH.

We find that SSDH is applicable under three key factors. 1) The participant is unfamiliar with another one. In the CRAHNs, an SU needs the cooperation with other SUs to sense the status of the primary spectrum, but he worries about other SUs might be vicious or malicious. It is important to establish trust between two parties for a specific action, especially when the SU is not sure about the trust of other SUs. 2) Sensing data are unprotected. None of effect cryptography technologies have been considered to protect sensing data in CRAHNs. 3) Sensing data are transparent during transmission. The topology of CRAHNs 
graph is meshed, and all SUs also act as routers forwarding incoming messages to neighbors. A message reaches the desired SU after going through a number of SUs. Hereby, sensing data can be hijacked easily.

The threats of SSDH mainly refer to four aspects, as shown in Fig.2.

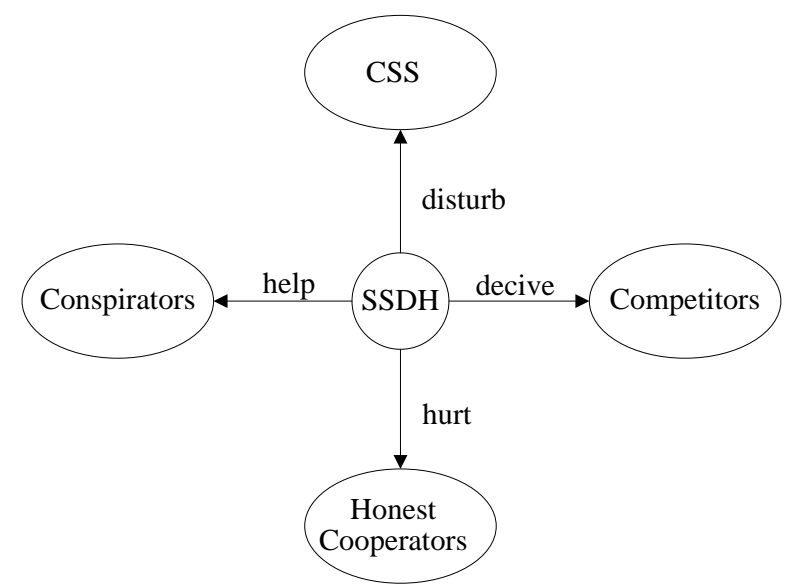

Fig. 2 Threats of SSDH .

- Disturbing CSS at a relatively low cost. SSDH attackers mislead the initiator SU make a wrong decision via CSS, in order to gain exclusive access to the target spectrum or give interference to PUs. Compared with SSDF attack, SSDH needs less cost to achieve the similar goals.

- Hurting honest cooperators. Cooperators are the SUs who play the role of sharing sensing data. Without loss of generality, we pay attention to SSDH attackers in this paper and assume that all cooperators share honest sensing data. However, the trust value of these honest cooperators will be reduces, when SSDH attackers hijack and tamper with their sensing data during transmission.

- Helping the conspirators of SSDH attackers. To intensify the power of SSDH, such attackers also help with each other to hijack sensing data to indicate the primary spectrums are in use, although it is unused. Thus, one of conspirators can gain exclusive access to the target primary spectrum.

- Deceiving the competitors of SSDH attackers. The primary spectrums are very finite in a CRAHN. To monopolize the limited primary spectrums, SSDH attackers should always indicate the primary spectrums are in use. In this case, other SUs make a wrong decision that PUs are present and will give up their spectrum opportunities.

In a CRAHN, the SSDH attack strategy can be constructed by four procedures, as shown in Fig.3.

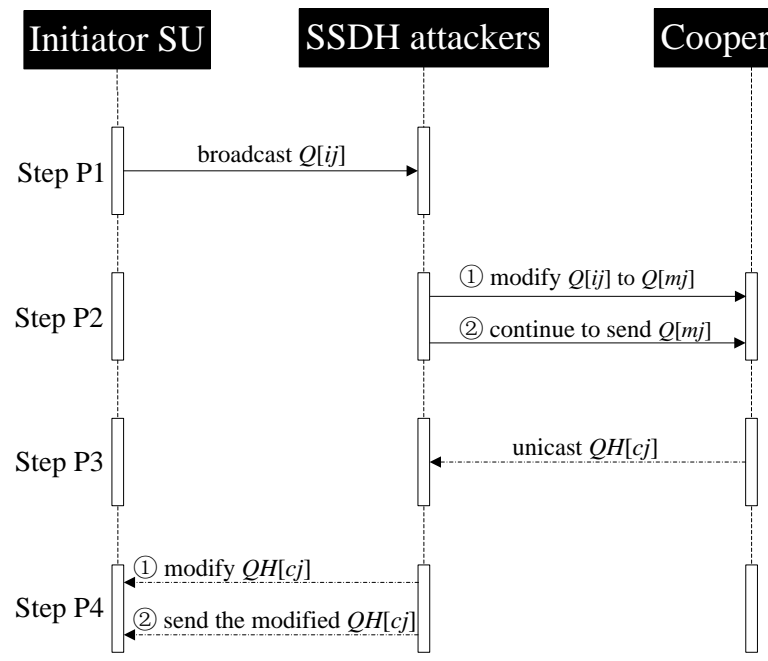

Fig.3 SSDH attack strategy.

Step P1. An initiator $\mathrm{SU}$ (such as $\mathrm{SU}_{i}$ ) broadcasts a Query message $Q[i j]=\left(S U_{i}, P U_{j}, C S S_{i j}, S T_{i j}\right)$ 
related on one PU's spectrum (such as $\mathrm{PU}_{j}$ ). The query is labeled by a unique ID of two participators that can be used by the recipients to detect where the Query comes from. $C S S_{i j}$ indicates that $S U_{i}$ is looking for the status of $P U_{j}$ spectrum. $S T_{i j}$ is the sensing time for $C S S_{i j}$ initiated by $\mathrm{SU}_{i}$.

Step P2. Assuming $\mathrm{SU}_{m}$ is one of SSDH attackers who wants to manipulate the CSS process. By playing the role of router, $\mathrm{SU}_{m}$ monitors all the Query messages via him. When $Q[i j]$ appears, $\mathrm{SU}_{m}$ changes the ID of $\mathrm{SU}_{i}$ into $\mathrm{SU}_{m}$, that is $Q[i j] \stackrel{\text { modified }}{\longrightarrow} Q[m j]$. In this case, $\mathrm{SU}_{m}$ would receive all the QueryHit messages corresponding to the tampered $Q[m j]$.

Step P3. Assuming $\mathrm{SU}_{c}$ is one of cooperators who has the ability to sensing $P U_{j}$ spectrum. $\mathrm{SU}_{c}$ answers with a QueryHit unicast message $Q H[c j]=\left(\mathrm{SU}_{m}, P U_{j}, \mathrm{SU}_{c}, s s d_{c j}, S T_{k j}\right)$ to $\mathrm{SU}_{m} . s s d_{c j}$ is the sesing data provided by $\mathrm{SU}_{c}$. $S T_{c j}$ is the time for $\mathrm{SU}_{c}$ sensing $\mathrm{PU}_{j}$.

Step P4. Upon reception of $Q H[c j], \mathrm{SU}_{m}$ tampers with $s s d_{c j}$ for his purpose, and changes $\mathrm{SU}_{m}$ back to $\mathrm{SU}_{i}$. At last, $\mathrm{SU}_{m}$ sends the tampered $Q H[c j]$ to $\mathrm{SU}_{i}$.

To further evaluate the power of SSDH attack, four parameters related to CRAHNs should be taken into account.

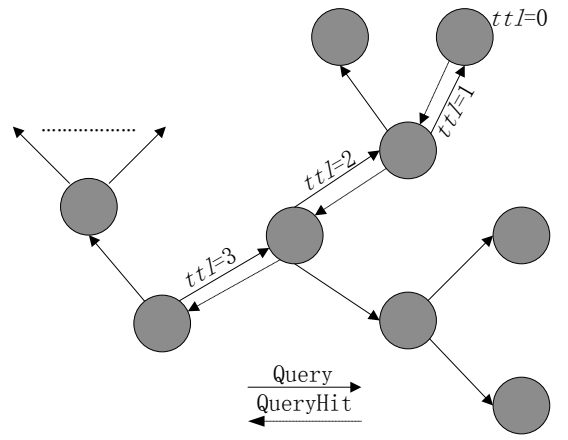

Fig.4 Sensing data aggregation at $n e i=2$.

- Neighbors (nei). In CRAHNs, each SU is linked dynamically to a small number of neighbors. So, an initiator SU broadcasts the Query message to all his neighbors, and then they forward the Query message to their neighbors until reaching the destination.

- Time-to-live $(t t l)$. To reduce network congestion, all the messages exchanged on the network are characterized by a given $t t l$. On passing through an SU, the $t t l$ of a Query message is decreased by one. When the $t t l$ reaches zero, the message is dropped.

- Distance (dis). Upon hijacking a Query message, the message has been through a few SUs from the initiator SU to an SSDH attacker $\left(\mathrm{SU}_{m}\right)$. We define the $t t l$ consumed from the initiator $\mathrm{SU}$ to $\mathrm{SU}_{m}$ as dis. In Fig.4, we fix dis as 1 and $t t l$ as 3. The amount of SUs forwarding the tampered Query message $\left(Q_{m}\right)$ for $\mathrm{SU}_{m}$ can be calculated as $2^{1}+2^{2}=6$. This conclusion can be further extended as

$$
Q_{m}=\sum_{n} n e i^{n}, 1 \leq n \leq t t l-d i s
$$

- Probability (pro). This parameter denotes the probability of responding the tampered Query message by $\mathrm{SU}_{m}$. Obviously, cooperators will respond or not respond to the Query, so pro $\mathrm{B}\left(Q_{m}, p\right)$. $p$ is the probability of responding to the Query for cooperators. Assuming the number of cooperators who respond to the Query is $r$, pro can be evaluated as

$$
\text { pro }=\left(\begin{array}{l}
Q_{m} \\
r
\end{array}\right) p^{r}(1-p)^{N_{m}-r}, 0 \leq r \leq N_{m}
$$

It can be deduced that the expectation value of the binomial distribution is given by: $\mathrm{E}[$ pro $]=p Q_{m}$. Thus, the attack power of $\mathrm{SU}_{m}$ can be describe as

$$
P_{m}=p Q_{m}
$$

Let $\Phi$ denote the set of SSDH attackers in a CRAHN, the power of SSDH attack can be calculated as

$$
P^{S S D H}=\sum_{m} P_{m}, S U_{m} \in \Phi
$$


For three typical CSS fusion rules, SSDH attackers will succeed under the following case

$$
S S D H \stackrel{\text { attack }}{\longrightarrow} \mid \begin{aligned}
& P^{S S D H} \geq 1, " \text { OR" succeed } \\
& P^{S S D H} \geq N, " A N D " \text { succeed } \\
& P^{S S D H} \geq N / 2, " \text { Majority" succeed }
\end{aligned}
$$

$N$ denotes the number of sensing data collected for an initiator SU.

\section{Proposed defense scheme}

We develop a two-level defense scheme called GSSD including IBC signature-verification and neighbor monitor, which aims to suppress SSDH attack by guarding spectrum sensing data transmission.

\subsection{Defense: IBC signature-verification}

To protect sensing data from being tampered, a direct method is to adopt the public key cryptography (PKC). Unfortunately, there is not a central authority to manage key exchange and PKC signatures (e.g., RSA [18]) require heavy transmission overhead and long execution time. Therefore, PKC may not be a good solution in CRAHNs.

In the identity-based cryptography (IBC) [10], a user's public key is derived from a user's identity information such as e-mail address or telephone number, and thus eliminate the requirement for public-key certificate transmission and storage. Here, we utilize the IBC to design the GSSD scheme. To show how the signature-verification works for sensing data transmission, we employ the Chch scheme [19] to sign all sensing data, which is viewed as one of the most efficient identity-based signatures.

Considering that most of the IBC signatures are based on pairing techniques, we illustrate the signature verification algorithm as follows.

- Setup. $Z$ is a cyclic additive group of prime order $t$ on an elliptic curve $E$ and $Q$ is a generator of $Z$. The pairing $\hat{e}$ is a map from $Z \times Z$ into $Z_{T}$ where $Z_{T}$ is a cyclic multiplicative group of the same order $t$ on $E$. Let $H_{1}:\{0,1\}^{*} \times Z \rightarrow Z_{T}^{*}$ and $H_{2}:\{0,1\}^{*} \rightarrow Z$. An initiator SU selects a random integer $s$ in $Z_{T}^{*}$ as the query private key and issues this value. Likewise, the initiator SU computes $Q_{p u b}=t Q$ as the query public key.

- Sign. For a cooperator $\mathrm{SU}_{c}, H_{2}\left(\mathrm{ID}_{c}\right)$ and $\mathrm{sH}_{2}\left(\mathrm{ID}_{c}\right)$ are his public key and private key, respectively. To sign $s s d_{c}, \mathrm{SU}_{c}$ randomly chooses an integer $q$ in $\square_{t}^{*}$ and generates a signature $\sigma_{c}=\left(U_{c}, V_{c}\right)$ where $U_{c}=q H_{2}\left(\mathrm{ID}_{c}\right)$ and $V_{c}=s H_{2}\left(\mathrm{ID}_{c}\right)\left(q+H_{1}\left(s s d_{c}, U_{c}\right)\right)$. $\mathrm{ID}_{c}$ denotes the identity number of $\mathrm{SU}_{c}$.

- Verify. Upon receiving $\sigma_{c}$ and $s s d_{c}$, the initiator SU computes $h_{c}=H_{1}\left(s s d_{c}, U_{c}\right)$ and verifies the signature. If $\hat{e}\left(Q, V_{c}\right)=\hat{e}\left(Q_{p u b}, U_{c}+h_{c} H_{2}\left(I D_{c}\right)\right), \sigma_{c}$ is a valid signature.

The following result ensures the correctness of the verify operation.

$$
\begin{aligned}
\text { Proof: } & \hat{e}\left(Q, V_{c}\right)=\hat{e}\left(Q_{p u b}, U_{c}+h_{c} H_{2}\left(I D_{c}\right)\right) \\
\hat{e}\left(Q, V_{c}\right)= & \hat{e}\left(Q, s H_{2}\left(I D_{c}\right)\left(q+H_{1}\left(s s d_{c}, U_{c}\right)\right)\right) \\
= & \hat{e}\left(s Q, q H_{2}\left(I D_{c}\right)+H_{1}\left(s s d_{c}, U_{c}\right) H_{2}\left(I D_{c}\right)\right) \\
= & \hat{e}\left(Q_{p u b}, U_{c}+h_{c} H_{2}\left(I D_{c}\right)\right)
\end{aligned}
$$

\subsection{Defense: neighbor monitor}

In the first level defense, we can detect whether sensing data have been tampered by SSDH attackers. To defend agaainst SSDH attack, only the first level defense is not enough. There are two other problems should be addressed: 1) is possible to correct the tampered sensing data during transmission? and 2) how to encourage SSDH attackers not to tamper with sensing data again? 


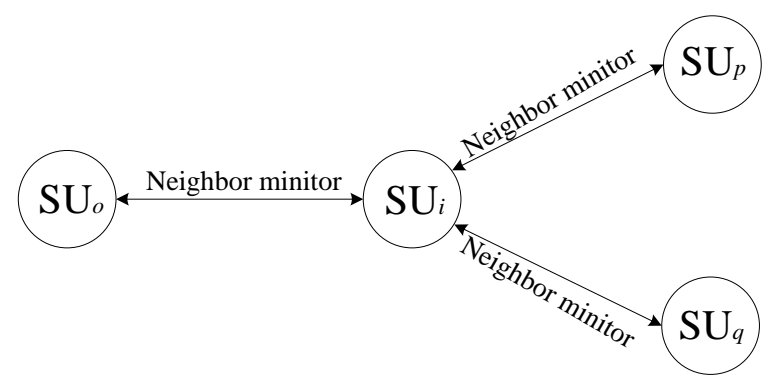

Fig.5 Example of neighbor monitor among SUs in CRAHNs.

Considering no central authority in CRAHNs, we introduce the idea of neighbor monitor to design the second level defense. As shown in Fig.5, assume a router $\mathrm{SU}_{i}$ has three neighbors: $\mathrm{SU}_{o}, \mathrm{SU}_{p}$ and $\mathrm{SU}_{q}$. When $\mathrm{SU}_{i}$ forwards QueryHit received from his neighbors such as $\mathrm{SU}_{p}$, he should firstly verify $\left(s s d_{c}, \sigma_{c}\right)$ and update the neighbor tie value $n t_{i p}$ generated from $\mathrm{SU}_{i}$ to $\mathrm{SU}_{p}$, and then forward QueryHit to $\mathrm{SU}_{o}$. This process can be performed by Procedure 1 .

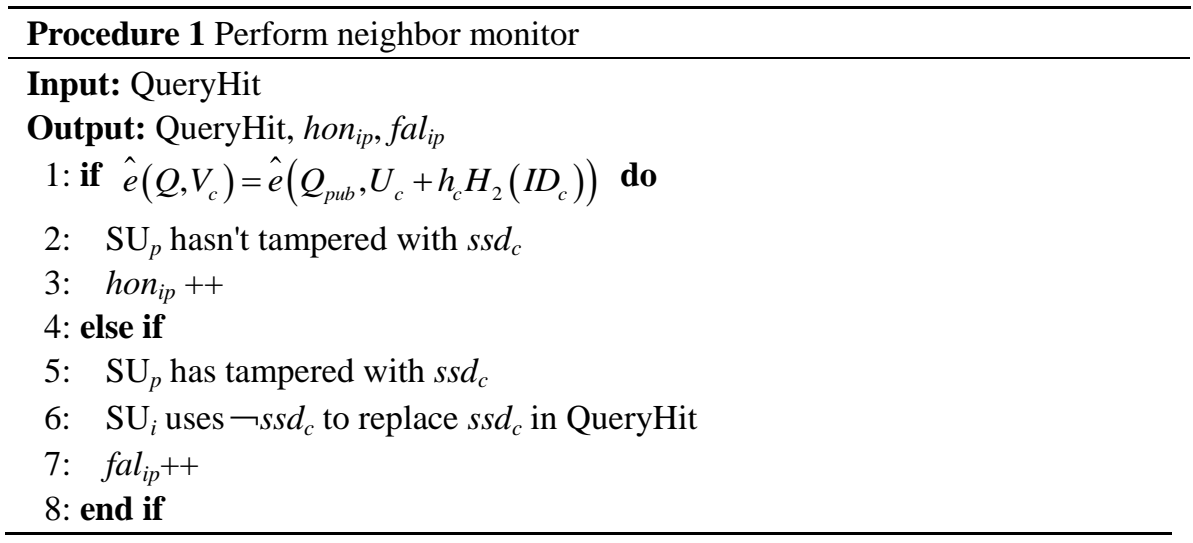

It can be found that it is possible in Procedure 1 to correct the tampered $s s d_{c}$. This is because $s s d_{c}$ is a binary variable $\{0,1\}$, and it is possible to correct $\operatorname{ss} d_{c}$ when $e\left(Q, V_{c}\right) \neq e\left(Q_{p u b}, U_{c}+h_{c} H_{2}\left(I D_{c}\right)\right)$. Specially, for $s s d_{c}=1, \neg s s d_{c}=0$.

The function of neighbor monitor is to make each SU mutually monitor his neighbors when forwarding spectrum sensing data in CRAHNs. That is, each SU pays attention to his neighbors' forwarding behaviors. When an $\mathrm{SU}$ such as $\mathrm{SU}_{i}$ receives the tampered $s s d_{c}$ from the previous neighbor $\mathrm{SU}_{p}$, he would better correct it. $\mathrm{SU}_{i}$ also has the right not to correct the tampered $s s d_{c}$. But, if not, it will affect the neighbor tie value $n t_{o i}$ generated from the next neighbor $\mathrm{SU}_{o}$ to $\mathrm{SU}_{i}$. Except for inspiring to correct, another reason for this is that such irresponsible behavior can facilitate the reproduction of SSDH attackers. So, SU $U_{i}$ should pay the price for his irresponsible behavior.

Here, neighbor tie is the key to maintain neighbor monitor. Concretely, once an SU often forwards honest sensing data, he would get a good neighbor tie value from his neighbors. Or else, he would get a bad neighbor tie value. With a good neighbor tie value, this SU can request his neighbors to help him easily.

In our scheme, we can find that each SU may behave two types of forwarding results: honest or false. Based on this, we can initialize neighbor tie by two factors: the number of honest forwarding (hon) and the number of false forwarding $(f a l)$. Recently, one of the most popular designs to evaluate binary input (i.e., positive or negative) is based on beta function. It first counts the number of honest and false behaviors a user has conducted, and then calculates a value with beta probability density functions (PDF) denoted by $\operatorname{Beta}(\alpha$, $\beta)[20]$.

$$
\operatorname{Beta}(q \mid a, b)=\frac{\mathrm{G}(a+b)}{\mathrm{G}(a) \mathrm{G}(b)} q^{a-1}(1-q)^{b-1}
$$


where $\theta$ is the probability of sensing behaviors, $0 \leq \theta \leq 1, \alpha>0, \beta>0$.

Take example for $\mathrm{SU}_{i}$ and $\mathrm{SU}_{p}$, hon $i p$ and $f a l_{i p}$ denote the number of honest forwarding (forwarding honest spectrum sensing data) and false forwarding (forwarding the tampered spectrum sensing data) performed by $\mathrm{SU}_{p}$ to $\mathrm{SU}_{i}$. The neighbor tie value $n t_{i p}$ can be evaluated with beta function as: $n t_{i p}=\operatorname{Beta}\left(h_{o n} n_{i p}+1, f_{i p}+1\right)$. Without any prior observations, $h_{o n}{ }_{i p}=f \mathrm{l}_{i p}=0$ and hence, $n t_{i p}=\operatorname{Beta}(1,1)$.

Consider the case $\Gamma(x)=(x-1)$ ! when $x$ is an integer [21]. It can be deduced that the expectation value of the beta function is given by: $\mathrm{E}[\operatorname{Beta}(\alpha, \beta)]=\alpha /(\alpha+\beta)$. Thus, $n t_{i p}$ can be described is as follows:

$$
n t_{i p}=\frac{1+\text { hon }_{i p}}{2+\text { hon }_{i p}+f a l_{i p}}
$$

However, there is a fatal flaw in Eq.(3) that only the malicious routers with continuous SSDH behaviors can be detected. If malicious routers are engaged in honest or false forwarding alternately, they would maintain a high neighbor tie value. To suppress such alternate behavior, the punishment to false forwarding is essential to evaluating the neighbor tie value. In the GSSD scheme, the additive punishment factor is introduced to attenuate the neighbor tie value of malicious routers. For $\mathrm{SU}_{i}$, his additive punishment factor $\lambda_{i p}$ corresponding to $\mathrm{SU}_{p}$ can be calculated as

$$
l_{i p}=\frac{f a l_{i p}}{1+\text { hon }_{i p}}
$$

For $f_{a l} l_{i p}=0, \lambda_{i p}$ will not be active. The more $\mathrm{SU}_{p}$ often lauches SSDH attack and forwards the tampered spectrum sensing data to $\mathrm{SU}_{i}$, the lower neighbor tie value he will get. To obtain a higher $n t_{i p}$, $\mathrm{SU}_{p}$ should refuse to SSDH attack and forward honest spectrum sensing data, resulting in the increase of $h_{o n}$ ip wich can boost the growth of $n t_{i p}$.

With $\lambda_{i p}, n t_{i p}$ can be further evaluated as

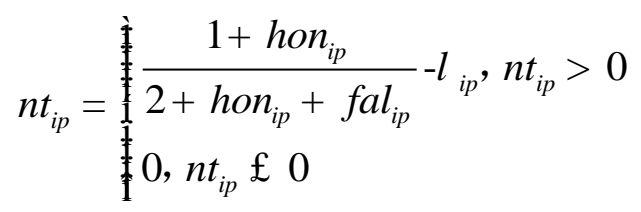

The goal of using neighbor tie value is to isolate SSDH attackers. If an SU holds a bad neighbor tie value, as punishment, his neighbors will refuse to forward his CSS request. That is, an SU with a bad neighbor tie value is difficult to get CSS help from cognitive ad hoc network. Take $\mathrm{SU}_{i}$ as an example again, when $\mathrm{SU}_{i}$ broadcasts a CSS Query message to his neighbors, Procedure 2 is performed by his neighbors. Let $N_{i}$ denote the set of $\mathrm{SU}_{i}$ 's neighbors and $\delta$ is the threshold of neighbor tie value.

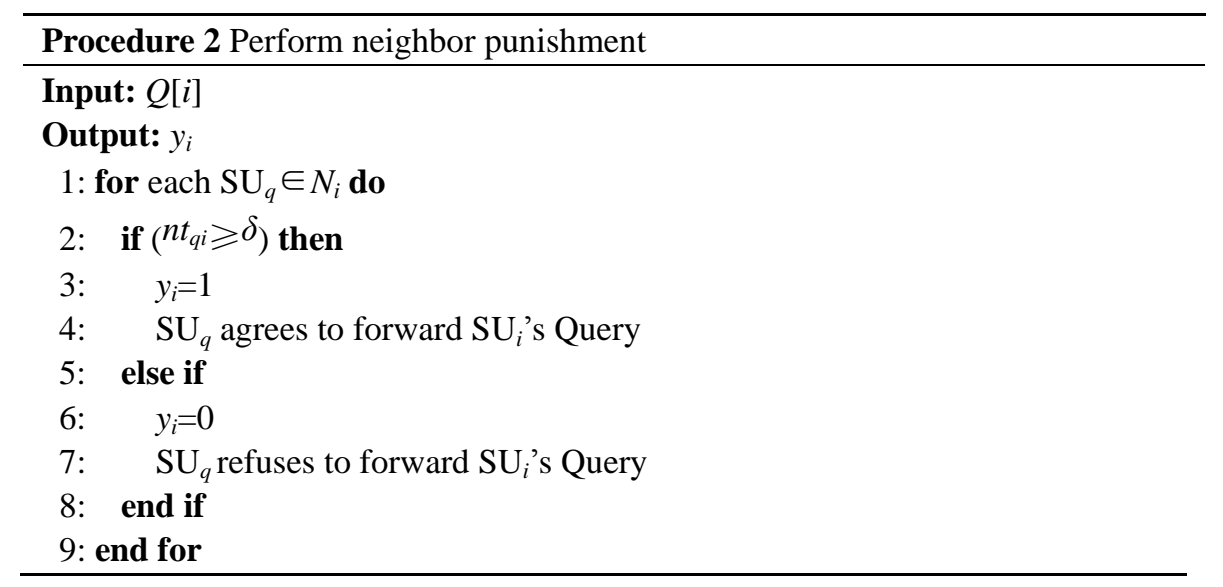

\subsection{Secure transmission strategies with GSSD}

By introducing the GSSD scheme, we can secure spectrum sensing data transmission against SSDH 
attack by five secure strategies, as shown in Fig.6.

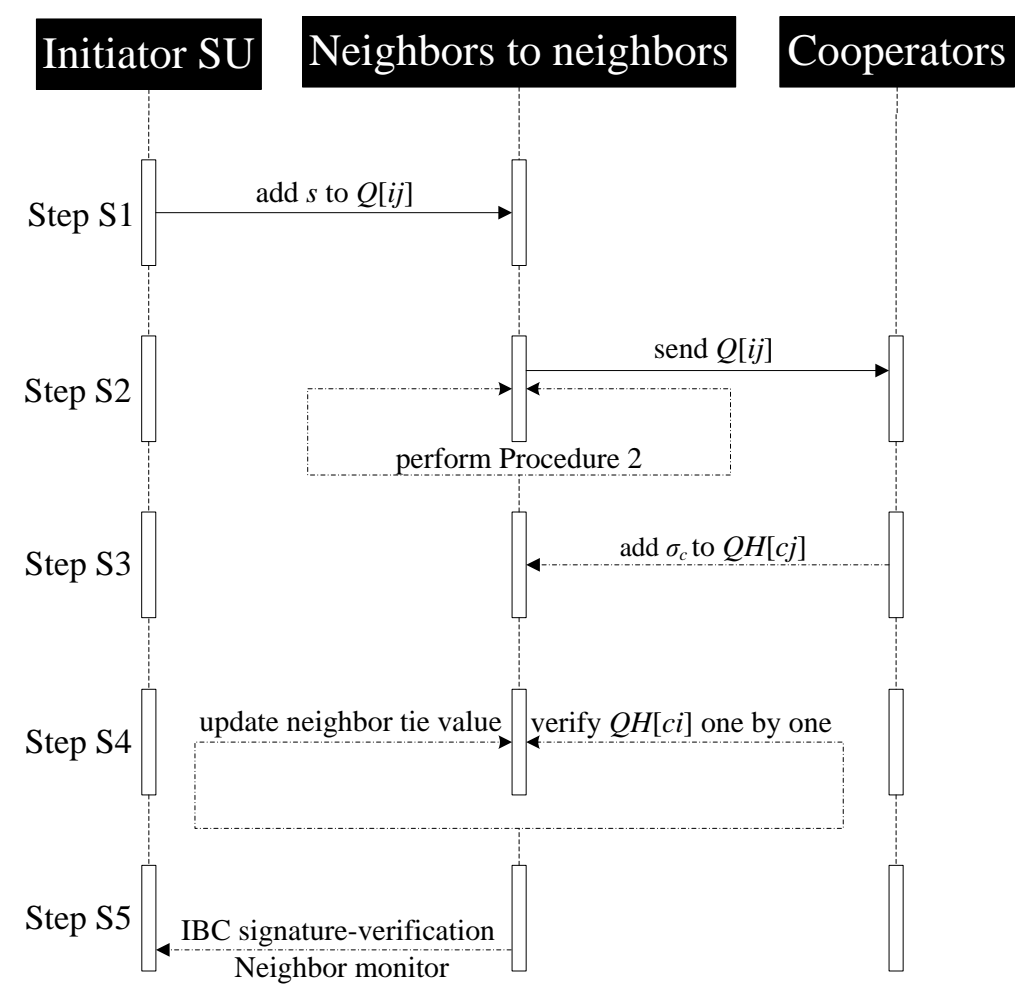

Fig.6 Secure strategies during sensing data transmission.

Step S1. $\mathrm{SU}_{i}$ broadcasts a CSS Query message $Q[i j]=\left(S U_{i}, P U_{j}, C S S_{i j}, S T_{i j}\right.$, $\left.s\right)$ to his neighbors where $s$ is the query private key .

Step S2. $\mathrm{SU}_{i}$ 's neighbors perform Procedure 2 to decide to whether to forward $Q[i j]$.

Step S3. A cooperator such as $\mathrm{SU}_{c}$ answers the CSS Query with a QueryHit unicast message $Q H[c j]=($ $\left.\mathrm{SU}_{i}, P U_{j}, \mathrm{SU}_{c}, s s d_{c j}, S T_{c j}, \sigma_{c}\right) . \sigma_{c}$ is $\mathrm{SU}_{c}$ 's signature on $s s d_{c}$.

Step S4. During the transmission of $Q H[c i]$, routers (neighbors to neighbors) verify $Q H[c i]$ one by one using IBC signature-verification and update their neighbors' neighbor tie value.

Step S5. Upon receiving a set of $Q H[c j]$ messages, the IBC signature-verification and neighbor monitor are also triggered by $\mathrm{SU}_{i}$.

The security analysis of these strategies can be discussed as follows.

- In the proposed GSSD scheme, the signature $\sigma_{c}=\left(U_{c}, V_{c}\right)$ is actually a one-time identity-based signature. Even though SSDH attackers obtain $s s d_{c}$ and $I D_{c}$, it is infeasible to forge a valid signature. This is because they cannot know the random integer $q$ to compute $U_{c}$.

- Even if SSDH attackers tamper with $s s d_{c}$, it is still difficult to cheat other routers since $\sigma_{c}$ becomes a invalid signature by $h_{c}=H_{1}\left(s s d_{c}, U_{c}\right)$.

- Neighbor monitor inspires malicious SUs to give up their SSDH behaviors in order to get high neighbor tie value. With a lower neighbor tie value, their neighbors will refuse to forward their any Query messages.

\section{Performance analysis}

\subsection{Simulation Setup}

Computer simulations are performed to further analyze SSDH attack and show the performance of its defense scheme-GSSD. The simulation elements are shown in table 1. 
Table 1 Description of simulation elements

\begin{tabular}{clc}
\hline \hline Paramater & Description & Default \\
\hline$N_{P}$ & Number of PUs in the network & 3 \\
$N_{S}$ & Number of SUs in the network & 60 \\
$n e i$ & Number of neighbors & 4 \\
$t t l$ & Time-to-live & 6 \\
$d i s$ & Distance between an initiator SU and SSDH attackers & $1 \sim 6$ \\
$n t_{\text {threshold }}$ & Threshold of neighbor tie value & 0.8 \\
\hline
\end{tabular}

The simulations initiate by cycle-based fashion. At each cycle, all SUs are selected to perform CSS actions with each other randomly. After a few cycles, a neighbor tie based network topology is gradually formed by neighbor monitor. SUs then use it to perform CSS actions at each cycle, and update the neighbor tie value on their neighbors.

\subsection{Simulation Results}

One of main goals of SSDH is to hurt or reduce the trust of honest cooperators, which will make their trust value occurs errors and deviate the real trust. A higher trust errors indicates lower accuracy in the calculation of trust value. The trust errors are specified by:

$$
T E=\frac{1}{P_{S}} \stackrel{P_{S}}{P_{j=1}} \sqrt{\frac{1}{T_{j}^{\prime}}\left(T_{j}^{\prime}-T_{j}\right)^{2}}
$$

where $T_{j}{ }_{j}$ and $T_{j}$ are the actual and measured trust value of $j$, respectively.

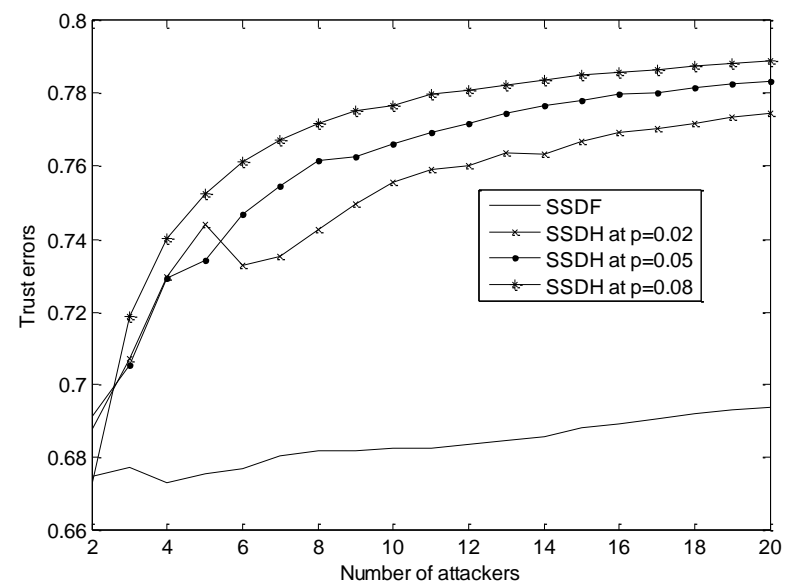

Fig.7 Trust errors with respect to SSDH and SSDF.

In the simulation of trust errors, the real trust value is randomly assigned. For honest cooperators, their real trust value is in the interval $(0.5,1]$. As shown in Fig.7, both SSDH and SSDF can cause the augment of trust errors with the increase of attackers. With a small responding probability $(p)$, such as $p=0.02$, the trust errors caused by SSDH is bigger than SSDF as the same number of attackers. The reason is that an SSDH attacker can tamper with several sensing data during the transmission while an SSDF attacker can only fake a sensing datum. Generally, the number of attackers denotes the cost of attack. Compared with SSDF attack, SSDH needs less cost to achieve the similar goals. We can also find that larger trust errors can be caused by higher responding probability at SSDH attack. Without loss of generality, in the following simulations, $p$ is set to 0.05 .

To evaluate the strengthen of attack, we compare SSDH and SSDF in terms of their attack success ratio. Then two attack patterns are simulated, namely, always-free and always-busy. In the always-free attack pattern, attackers fake sensing data that PU signals are absent to deceiving their competitors. In the always-busy attack pattern, attackers fake sensing data that PU signals are present to help their conspirators. 


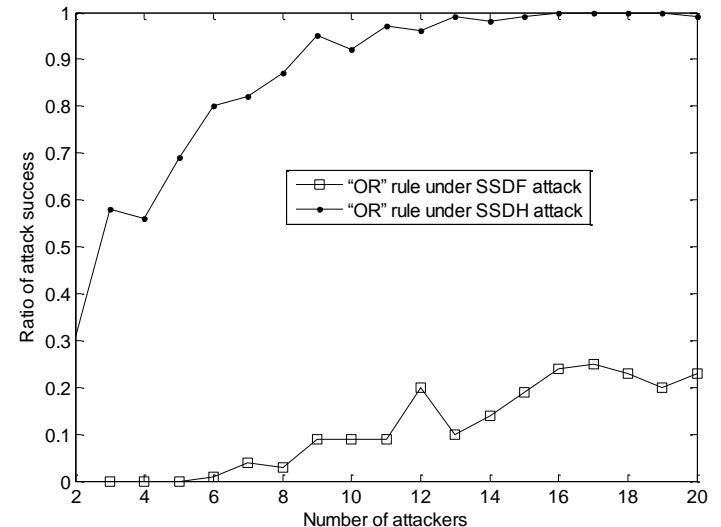

(a) always-free

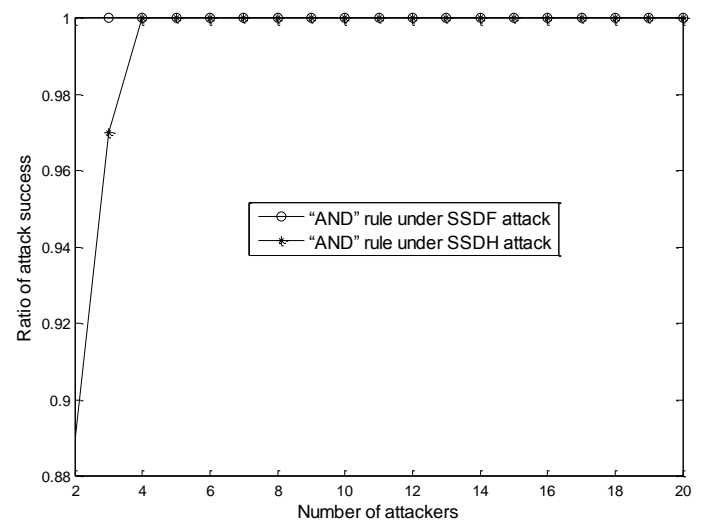

(c) always-free

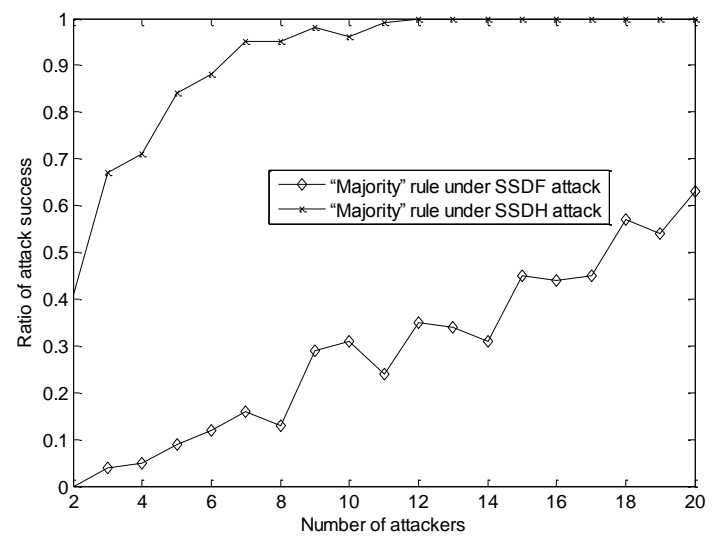

(e) always-free

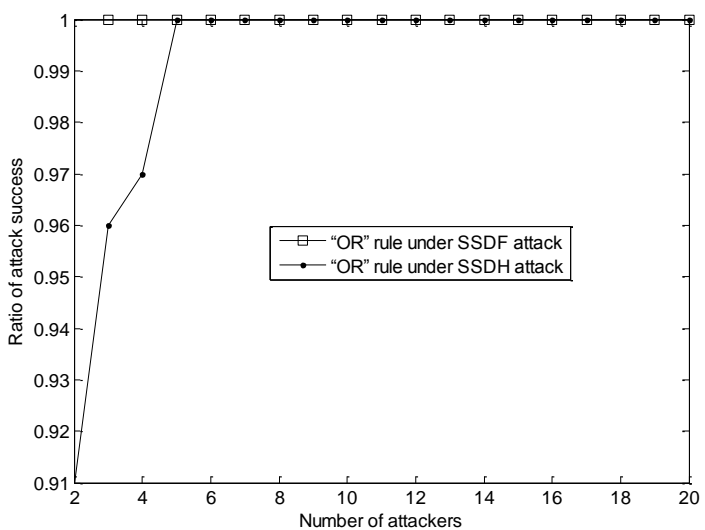

(b) always-busy

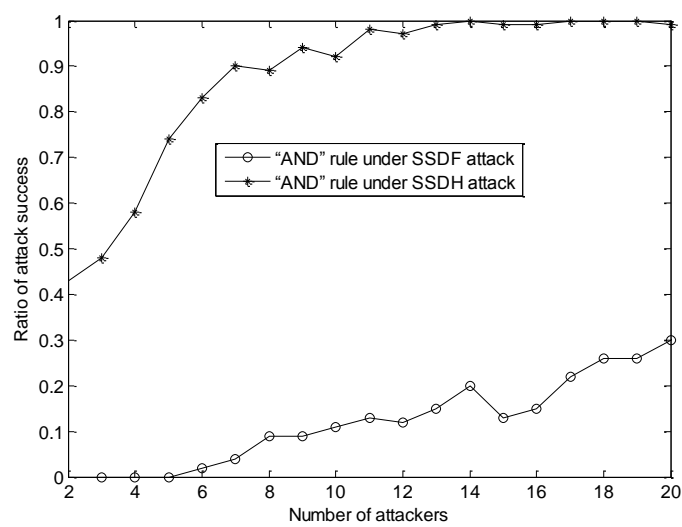

(d) always-busy

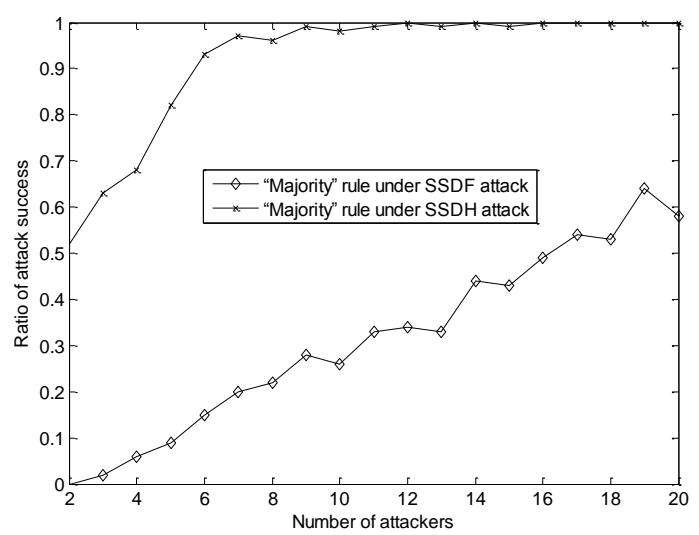

(f) always-busy

Fig.8 SSDH vs. SSDF at attack success rate.

As shown in Fig.8, SSDH can get higher attack success ratio than SSDF against the "AND", "OR" and "Majority" rule in some situations. In the always-free attack pattern, the damage of attacks to the "AND" rule is biggest. The final decision under the "AND" rule is " 0 " so long as an attacker tampers with the sensing data as" 0 ". In the always-busy attack pattern, the damage of attacks to the "OR" rule is biggest. The final decision under the "OR" rule is " 1 " so long as an attacker tampers with the sensing data as" 1 ". It can be seen that the damage of attacks against the "Majority" rule amplifies with the number of attackers. The attackers can change the final decision when they become the majority. This is because the majority of sensing data are 
"0" under the "Majority" rule, the final decision is " 0 ". Similarly, the majority of sensing data are " 1 " under the "Majority" rule, the final decision is "1". From this figure, the "Majority" rule is a good choice to make a reliable final decision.

To defend against SSDH attack, the first important measure is to suppress trust errors. As shown in Fig.9, the GSSD scheme can reduce trust errors when the number of SSDH attackers is smaller. However, trust errors also augment with the increase of attackers. The reason is that an SSDH attacker can tamper with several sensing data during the transmission. For a large number of SSDH attackers, their powers will become more severe. From SSDH attackers to initiator SUs, the numerous tampered sensing data may pass through many neighbors. As long as one neighbor is unwilling to correct the tampered sensing data, the trust value of cooperators would be misled.

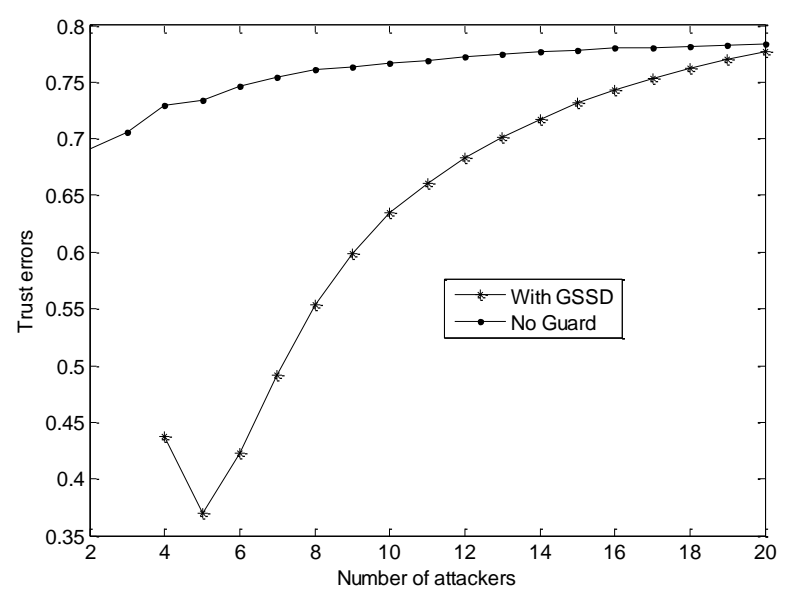

Fig.9 Trust errors with the guard of GSSD.

In fact, suppressing attack success ratio is the key to defending against SSDH attack. In GSSD, the IBC signature-verification scheme can identify the tampered sensing data. Meanwhile, neighbor monitor inspires malicious SUs to give up their SSDH behaviors in order to get high neighbor tie value. It can be seen in Fig.10, the GSSD scheme can suppress SSDH attack success ratio better under the "AND", "OR" and "Majority" rule. For the "OR" rule, only one false "1" data can make the final decision as "1". For the "AND" rule, only one false " 0 " data can make the final decision as " 0 ". Therefore, to make a reliable final decision, the "OR" rule is not a good choice at the always-busy attack pattern, and the "AND" rule is not a good choice at the always-free attack pattern.

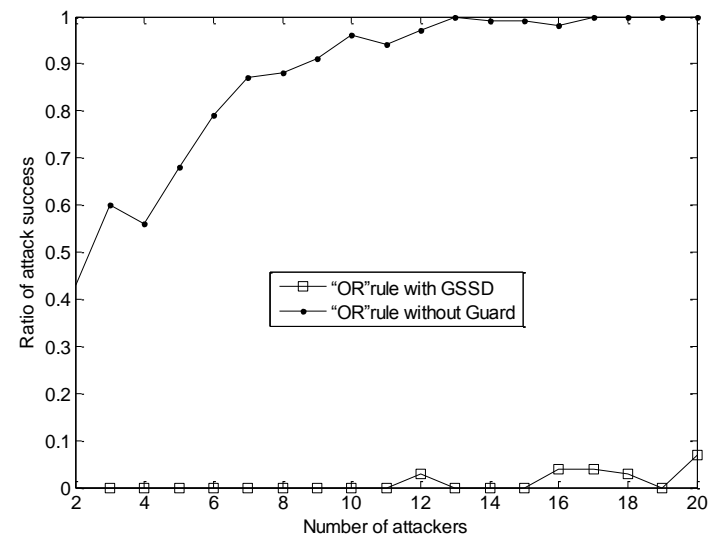

(a) always-free

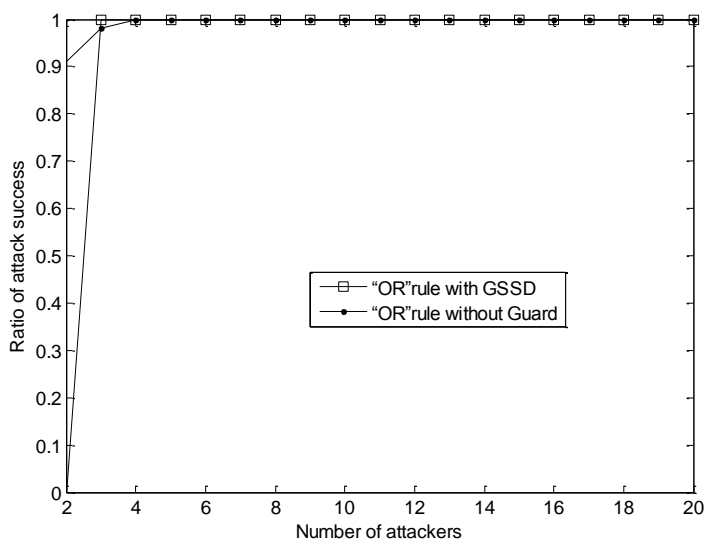

(b) always-busy 


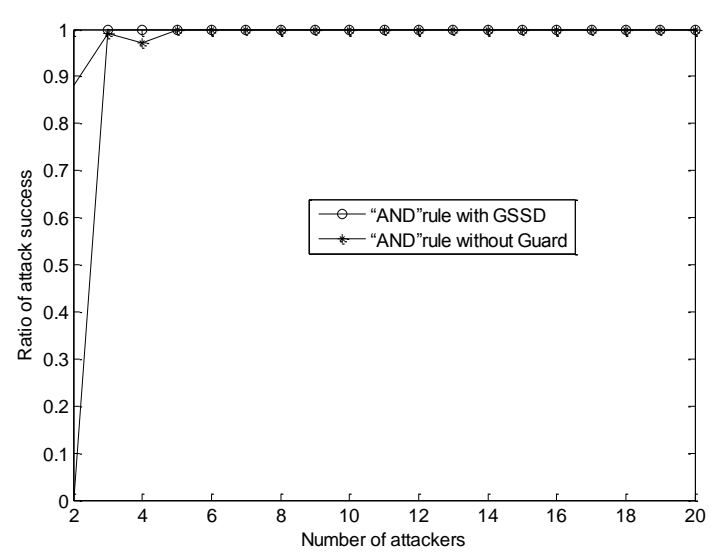

(c) always-free

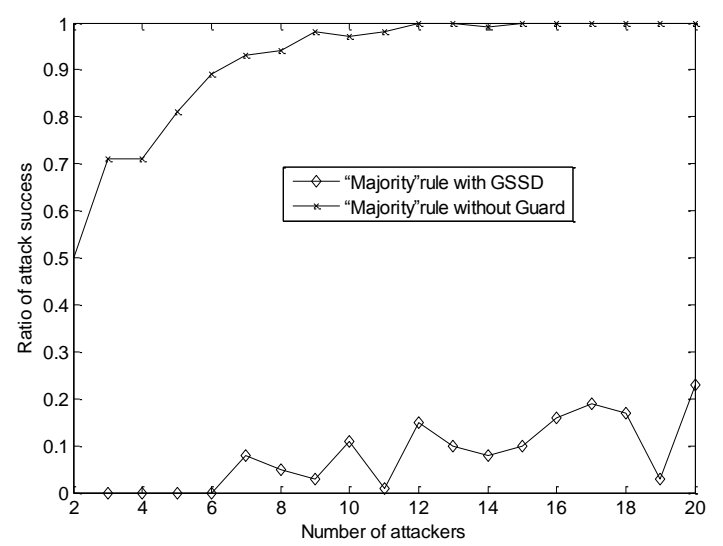

(e) always-free

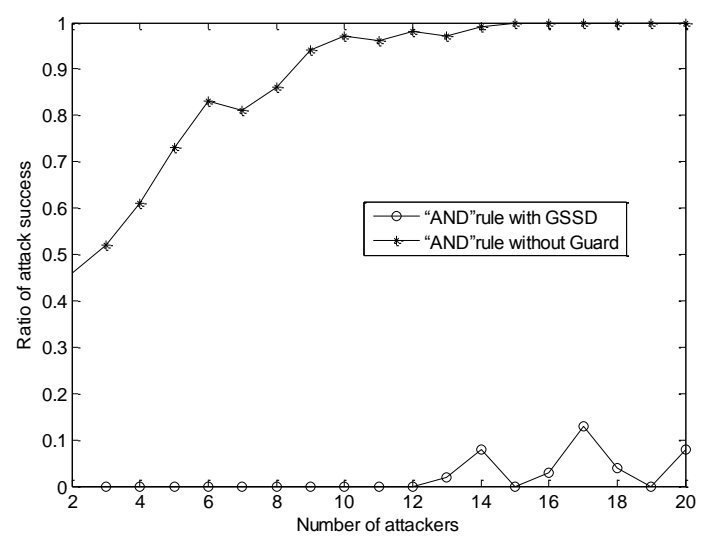

(d) always-busy

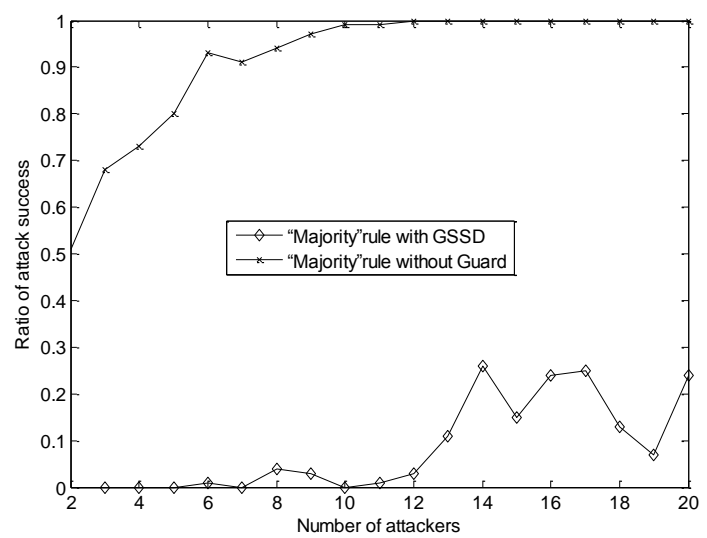

(f) always-busy

Fig.10 Suppressing SSDH attack success ratio.

\section{Conclusions}

Due to the nature of aggregating sensing data, CSS is becoming an attractive target for attackers. In this paper, we discover a novel attack, named SSDH, against CSS in CRAHNs. The SSDH attackers can disguise as routers to hijack and tamper with sensing data. Compared with SSDF attack, SSDH needs less cost to achieve the similar goals. We conduct an in-depth investigation on SSDH and propose a two-level defense scheme called GSSD from the design ideas of IBC signature-verification and neighbor monitor. The simulations show that GSSD can reduce trust errors when the number of SSDH attackers is smaller, and significantly reduce the SSDH attack success ratio.

\section{References}

[1] Federal Communications Commission, "Spectrum Policy Task Force," Rep. ET Docket no. 02-135, Nov. 2002

[2] Le The Dung and Beongku An. How Do Forwarding Schemes Influence the Multi-hop Connectivity in Cognitive Radio Ad-hoc Networks? The Seventh International Conference on Ubiquitous and Future Networks (ICUFN 2015), July 7-10, 2015, Sapporo, Japan.

[3] I. F. Akyildiz, W. Y Lee and K. R Chowdhury, "CRAHNs: cognitive radio ad hoc networks," Ad Hoc Networks, vol. 7, no.5, pp. 810-836, October, 2009.

[4] R. L Chen, J. M Park and Y. T Hou, "Toward Secure Distributed Spectrum Sensing in Cognitive Radio Networks," IEEE Communications Magazine, vol. 46, no. 4, pp. 50-55, April, 2008.

[5] T. Qin, H. Yu, C. Leung, "Towards a trust-aware cognitive radio architecture," ACM SIGMOBILE Mobile Computing and Communications Review. vol.13, no.2, PP.86-95, 2009. 
[6] Zeng K, Peng Q H, Tang Y X, "Mitigating spectrum sensing data falsification attacks in hard-decision combining cooperative spectrum sensing," Science China, vol.57, no.4, pp.1-9, 2014.

[7] J.Y Feng, G.Y Lu, H Chang, "Behave well: How to Win a Pop Vacant Band via Cooperative Spectrum Sensing," KSII Transactions on Internet and Information Systems, vol.9, no.2, pp.1321-1336, 2015.

[8] Q. Q Pei, B. B Yuan, L. Li, H.N Li, "A sensing and etiquette reputation-based trust management for centralized cognitive radio networks,” Neurocomputing, vol.101, pp.129-138, 2013.

[9] I. F. Akyildiz, B. F. Lo and R. Balakrishnan, "Cooperative spectrum sensing in cognitive radio networks: A survey," Physical Communication, vol. 4, no. 1, February, pp. 40-62, 2011.

[10] Shamir A. Identity-based cryptography. Wikipedia [2012-10-17]. http://en.wikipedia.org/wiki/ID-based_cryptography

[11] E. Peh, Y. C Liang, Y. L Guan and Y. G Zeng, Optimization of Cooperative Sensing in Cognitive Radio Networks: A Sensing-Throughput Tradeo View, IEEE Transactions on Vehicular Technology, vol. 58, no. 9, pp. 5294-5299, November, 2009.

[12] M. A Morid and M. Shajari, An enhanced e-commerce trust model for community based centralized systems, Electronic Commerce Research, vol. 12, no. 4, pp. 409-427, November, 2012.

[13] W. Fang, C. Zhang, Z. Shi, et al. BTRES: Beta-based Trust and Reputation Evaluation System for wireless sensor networks. Journal of Network \& Computer Applications, vol. 59, no. 1, pp. 88-94, 2016.

[14] A. Boukerche, Y. Ren and R. Pazzi. An adaptive computational trust model for mobile ad hoc networks, in Proc. the 5th International Conference on Wireless Communications and Mobile Computing, pp. 191-195, June 21-24, 2009.

[15] Y. Wang, G. Yin, Z. Cai, et al. A trust-based probabilistic recommendation model for social networks. Journal of Network \& Computer Applications, vol. 55, no. 9, pp.59-67, 2015.

[16] Z. Yan, P. Zhang, A.V Vasilakos. A survey on trust management for Internet of Things. Journal of Network \& Computer Applications, vol. 42, no. 3, pp. 120-134, 2014.

[17] H. Rif-Pous, M. Blasco and C. Garrigues, "Review of Robust Cooperative Spectrum Sensing Techniques for Cognitive Radio Networks,” Wireless Personal Communications, vol. 67, no. 2, pp. 175-198, November, 2011.

[18] Jonsson J and Kaliski B. Public-key cryptography standards \#1: RSA cryptography specifications version 2.1. Technical report RFC 3447, 2003. [2012-10-20] http://rfc-ref.org/RFC-TEXTS/3447/index.html

[19] Cha J C and Cheon J H. An identity-based signature from gap diffie-hellman groups. Proceeding of 6th International Workshop on Practice and Theory in Public Key Cryptography, January 6-8, 2003, Miami, FL, USA. Berlin, Heidelberg, German: Springer, 2003: 18-30.

[20] A. J $\phi$ sang, R. Ismail, The beta reputation system, Proc. the 15th Bled Electronic Commence Conference, pp.1-14, June 2002.

[21] Gamma function. http://en.wikipedia.org/wiki/Gamma function 